\title{
Puberté et sports de compétition chez l'adolescente
}

\author{
Panagiota (Nota) Klentrou \\ Département d'Education Physique et de Kinésiologie, Université Brock, Sainte Catherine, Canada
}

\section{Mots-clés}

Sportives, entraînement/croissance/maturation • Développement hormonal · Menstruations · Fonction reproductrice $\cdot$ Composition corporelle $\cdot$ Restriction diététique $\cdot$ Aménorrhée de la sportive

\section{Résumé}

Ces dernières décennies, la participation croissante de jeunes enfants à un entraînement physique intensif a été à l'origine de préoccupations quant aux effets potentiels de cette situation sur la croissance et la maturation. La puberté se caractérise chez l'être humain par d'importantes modifications hormonales responsables de la maturation physique et sexuelle. Un entraînement intensif avant la puberté, ainsi que les effets métaboliques potentiels du régime alimentaire adopté, peuvent altérer la fonction hypothalamo-hypophysaire, et le moment auquel l'entraînement athlétique débute a été impliqué à titre de facteur de retard de la ménarche et de la maturation sexuelle chez les sportives. D'autre part, certaines études ont suggéré qu'un retard de la ménarche est probablement dû à des facteurs génétiques. Les jeunes filles qui sont matures plus tardivement sélectionnent souvent elles-mêmes des sports qui favorisent une petite taille ou une grande minceur, ou sont recrutées par les entraîneurs pour ceux-ci. La composition corporelle a été également utilisée afin d'expliquer simultanément le retard de la ménarche et les irrégularités menstruelles observées chez les sportives de haut niveau. Une prévalence plus élevée des dysfonctions menstruelles a été décrite chez des adolescentes pratiquant des sports dépendant du poids, comparativement à celle observée avec d'autres activités sportives. Toutefois, comme récemment suggéré, il n'existe aucune relation directe de cause à effet entre la corpulence et la reproduction et, de fait, c'est la disponibilité de l'énergie, et non les tissus adipeux, qui régule la fonction reproductive chez la femme. Des recherches supplémentaires sont justifiées afin de mieux explorer cette interaction entre les modifications à court terme de la disponibilité de l'énergie et l'aménorrhée due au sport chez l'adolescente. Nous concluons que, en raison des nombreux facteurs influençant de façon avérée la ménarche et la menstruation, le rôle du seul entraînement physique à titre de facteur causal d'un retard pubertaire et d'irrégularités menstruelles chez les jeunes femmes sportives est encore mal défini. Des recherches comportant des études longitudinales sont nécessaires afin de déterminer si les différences de maturité observées entre des jeunes femmes sportives ou non résultent de la nature ou de la nutrition, et quel est l'équilibre entre ces deux facteurs.

Copyright $\odot 2006$ Nestec Ltd., Vevey/S. Karger AG, Basel

\section{Introduction}

La puberté est un événement important de la croissance et de la maturation humaine. Elle est associée à d'importantes modifications physiologiques et psychologiques et se caractérise par des adaptations neuroendocriniennes significatives. De ce fait, au cours de la puberté, le milieu hormonal d'une adolescente sportive passe par une série d'adaptations induites par le développement, en modification constante et provenant de l'entraînement.

Le nombre croissant d'adolescentes ayant participé à des compétitions sportives ces dernières décennies a suscité un intérêt accru pour la physiologie de la jeune femme pratiquant des activités physiques. La croissance et la maturation se caractérisent par une variation individuelle et, bien que sous contrôle génétique et neuroendocrinien, des facteurs environnementaux, dont le type, l'intensité et la durée de l'entraînement, peuvent également exercer une influence [1].

\section{KARGER \\ Fax +41613061234 E-Mail karger@karger.ch} www.karger.com

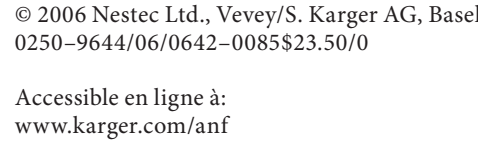

Nota Klentrou, $\mathrm{PhD}$

Department of Physical Education and Kinesiology

Brock University

St. Catharines, Ont. L6L 3M7 (Canada)

Tel. +1 905688 5550/ext. 4538, Fax +1 905688 8364, E-Mail nota.klentrou@brocku.ca 
A ce jour, les connaissances relatives aux effets de l'entraînement sportif sur l'enfant en croissance, particulièrement les filles, sont limitées en raison d'une difficulté de distinction entre les effets de l'entraînement et ceux de la croissance normale [1]. De ce fait, l'effet d'un entraînement intensif sur la croissance et la maturation des jeunes filles pratiquant des sports de compétition crée des actions physiologiques parallèles interactives et s'opposant parfois, avec des implications potentielles pour la santé et le développement des adolescentes. Bien entendu, la nutrition est également un important facteur à prendre en compte en raison de son rôle dans la maturation physique et le fonctionnement métabolique correct au cours de l'entraînement et des compétitions.

Le présent article examine la réponse à un entraînement intensif avant et pendant la puberté chez les jeunes filles. Il montre que, chez les sportives de haut niveau, et particulièrement chez les plus jeunes, l'association d'un entraînement intensif à une nutrition inadéquate résulte souvent en un retard de la ménarche et en des irrégularités menstruelles qui peuvent aboutir ultérieurement à des complications médicales. La discussion sera davantage centrée sur la question consistant à déterminer si les différences de maturité observées entre les jeunes femmes sportives ou non sont dues à la nature, à la nutrition ou à l'association des deux.

\section{Puberté et menstruation}

Le processus normal du cycle menstruel est régulé par une série d'interactions complexes entre les hormones hypophysaires et ovariennes. Ces hormones incluent l'hormone de libération de la gonadotrophine $(\mathrm{GnRH})$, secrétée par l'hypothalamus, et ciblent l'hypophyse afin que celle-ci libère l'hormone stimulant les follicules (FSH) et l'hormone lutéinisante (LH). Au cours de l'enfance, l'hypothalamus est sensible à de faibles concentrations d'œstrogènes, qui exercent un effet de rétroaction négative en inhibant l'activité hypothalamique. Le facteur précis déclenchant la puberté et l'apparition des premières menstruations n'est pas encore nettement élucidé; la survenue des événements apparentés, ainsi que le moment de la ménarche, varient fortement et sont influencés par la nutrition, l'hérédité, l'état général, le pourcentage de masse grasse, l'hormone de croissance et d'autres facteurs de croissance, ainsi que par la maturation de l'axe hypothalamo-hypophysaire, qui dépend elle-même de nombreux facteurs [2-4]. La puberté survient généralement chez les jeunes filles quand l'hypothalamus devient mature et moins sensible au rétrocontrôle négatif, ce qui favorise une augmentation de la sécrétion de GnRH par les cellules neurosécrétoires de l'hypothalamus, situation qui déclenche en retour le début de la sécrétion des hormones gonadotrophes, LH et FSH, par l'hypophyse antérieure. Des quantités suffisantes de FSH et de LH stimulent le dévelop- pement des follicules ovariens ainsi que celui des caractéristiques sexuelles secondaires et l'augmentation de la libération de stéroïdes sexuels féminins (œstrogène et progestérone) par les ovaires [2]. L’œstrogène et la progestérone continuent à augmenter pendant toute la durée de la puberté et jouent un rôle capital dans la maturation tant physique que sexuelle [3]. Tout au long des phases du cycle menstruel, la FSH favorise le développement du follicule et la sécrétion d'œstrogènes par les ovaires, tandis que la LH stimule l'ovulation et la sécrétion d'œstrogènes et de progestérone. Le cycle menstruel débute par la menstruation. A la phase folliculaire, qui correspond aux jours précédant l'ovulation, les concentrations de FSH et LH augmentent, et les œstrogènes deviennent prédominants. Après l'ovulation, au cours de la phase lutéale du cycle, la progestérone est plus importante que les œstrogènes tandis que l'utérus est préparé à une éventuelle grossesse.

Les sportives sont souvent préoccupées par le nombre de jours de menstruation et le volume du flux menstruel. Un cycle normal est souvent décrit comme d'une durée moyenne de 28 jours, mais, en réalité, un cycle de 28 jours ne survient que dans seulement $12,4 \%$ de l'ensemble des cycles. Des études de la durée «normale» de menstruations régulières ont montré des variations allant de 21 à 35 jours $[2,3]$. La durée menstruelle varie également considérablement, celle du flux habituel étant en moyenne de $2-8$ jours $[2,3]$.

Des auteurs ont suggéré que les œstrogènes (principalement leur composante estradiol) et la progestérone pouvaient être altérés par une activité physique intense. Des études ont montré que des modifications physiologiques survenaient à la phase folliculaire et à la phase lutéale lors de l'entraînement et des performances aérobies comme anaérobies [5].

\section{Survenue de la puberté chez les sportives}

Ces dernières décennies, la participation croissante de jeunes enfants à un entraînement physique intensif a suscité des préoccupations quant aux effets potentiels pour la croissance, la maturation et la fonction reproductive de ces sujets [6]. Il a été observé qu'un entraînement intense retarde l'apparition de la puberté chez les jeunes filles en altérant le développement hormonal normal $[7,8]$. Un retard de la poussée de croissance pubertaire, de l'âge de la ménarche et de la maturation squelettique ont été décrits chez de jeunes filles effectuant un entraînement sportif pendant au moins 15 heures/semaine [9]. La plupart des discussions ayant trait à l'influence potentielle de l'entraînement physique sur la maturation sexuelle ont été centrées sur l'accroissement de l'âge moyen à la ménarche, souvent décrit chez des adolescentes sportives [4, 10-19]. Le tableau 1 résume des données internationales concernant l'âge d'apparition 
Tableau 1. Age d'adolescentes sportives et non sportives à la ménarche (moyenne \pm écart type)

\begin{tabular}{|c|c|c|c|}
\hline Etude & Pays & Groupe de sport & Age à la ménarche, années \\
\hline Torstveit et & Norvège & Sportives de haut niveau $(\mathrm{n}=669)$ & $13,4 \pm 1,4^{*}$ \\
\hline Sundgot-Borgen [10], 2005 & & Témoins d'âge correspondant $(\mathrm{n}=607)$ & $13,0 \pm 1,3$ \\
\hline \multirow[t]{2}{*}{ Klentrou et Plyley [11], 2003} & Canada & Gymnastes rythmiques $(\mathrm{n}=30)$ & $13,6 \pm 1,2^{*}$ \\
\hline & & Témoins d'âge correspondant $(\mathrm{n}=40)$ & $12,3 \pm 0,8$ \\
\hline \multirow[t]{2}{*}{ Klentrou et Plyley [11], 2003} & Grèce & Gymnastes rythmiques de haut niveau $(\mathrm{n}=15)$ & $14,2 \pm 0,3^{*}$ \\
\hline & & Témoins d'âge correspondant $(\mathrm{n}=38)$ & $12,8 \pm 0,9$ \\
\hline \multirow[t]{3}{*}{ Dusek [12], 2001} & Croatie & Sportives entraînées avant la ménarche $(\mathrm{n}=34)$ & $13,8 \pm 1,4^{*}$ \\
\hline & & Sportives entraînées après la ménarche $(\mathrm{n}=33)$ & $12,6 \pm 1,0$ \\
\hline & & Témoins d'âge correspondant $(\mathrm{n}=96)$ & $13,0 \pm 1,2$ \\
\hline \multirow[t]{2}{*}{ Pigeon et coll. [13], 1997} & France & Ballerines $(n=97)$ & $13,5^{*}$ \\
\hline & & Témoins $(\mathrm{n}=30)$ & 12,0 \\
\hline \multirow[t]{2}{*}{ Constantini et Warren [14], 1995} & Israël & Nageuses $(n=69)$ & $13,8 \pm 0,2^{*}$ \\
\hline & & Témoins ( $\mathrm{n}=279)$ & $13,0 \pm 0,1$ \\
\hline \multirow[t]{2}{*}{ Lindholm et coll. [4], 1994} & Suède & Gymnastes artistiques $(\mathrm{n}=22)$ & $14,5 \pm 1,4^{*}$ \\
\hline & & Témoins $(\mathrm{n}=22)$ & $13,2 \pm 0,9$ \\
\hline Malina et coll. [15], 1994 & États-Unis & 7 sports - diverses sportives $(n=109)$ & $13,8 \pm 1,5$ \\
\hline \multirow[t]{3}{*}{ Hata et Aoki [16], 1990} & Japon & Sportives de haut niveau $(\mathrm{n}=40)$ & $13,5 \pm 1,3$ \\
\hline & & Sportives de collèges $(n=386)$ & $12,9 \pm 1,2$ \\
\hline & & Sportives d'écoles supérieures $(\mathrm{n}=253)$ & $12,6 \pm 1,1$ \\
\hline Calabrese [17], 1985 & États-Unis & Gymnastes artistiques $(\mathrm{n}=20)$ & 14,2 \\
\hline Calabrese et coll. [18], 1983 & États-Unis & Ballerines $(\mathrm{n}=25)$ & 14,3 \\
\hline \multirow[t]{2}{*}{ Malina [19], 1973} & États-Unis & Pratiquant l'athlétisme $(\mathrm{n}=66)$ & $13,6 \pm 0,2^{*}$ \\
\hline & & Témoins $(\mathrm{n}=30)$ & $12,2 \pm 1,6$ \\
\hline
\end{tabular}

de la première menstruation chez de jeunes femmes sportives comparativement à des témoins non sportives. Certaines études ont toutefois suggéré qu'un retard de la ménarche était dû à des facteurs génétiques, les jeunes filles plus tardivement matures choisissant souvent d'elles-mêmes des sports qui nécessitent une force musculaire relativement élevée et une petite stature, telle que la gymnastique [20]. Selon Kaprio et coll. [21], 37\% de la variance de l'âge à la ménarche a été imputable à des facteurs génétiques supplémentaires. De plus, la corrélation entre l'effet génétique additionnel sur l'âge à la ménarche et l'indice de masse corporelle (poids/taille ${ }^{2}$ ) a été de 0,57 , indiquant une proportion substantielle d'effets génétiques. Les données longitudinales limitées concernant des jeunes filles sportives comparativement à d'autres non sportives n'ont indiqué aucun effet de l'entraînement sur le moment d'apparition ou la progression de caractéristiques sexuelles secondaires, dont le développement des seins et de la pilosité pubienne [22].
Un entraînement intensif avant la puberté peut altérer la fonction hypothalamo-hypophysaire, et le moment du début de l'entraînement athlétique a été donc impliqué en tant que facteur d'un retard de la ménarche, souvent constaté chez les sportives qui débutent un entraînement à un jeune âge [23]. Selon Dusek [12], des sportives croates ayant débuté des activités physiques avant leur ménarche ont indiqué que celle-ci avait été significativement retardée $[13,8 \pm$ $1,4$ contre $12,6 \pm 1,0$ ans; $\mathrm{p} \leq 0,001)$. La composition corporelle a été également utilisée à titre d'explication des retards de menstruation et des irrégularités menstruelles chez des sportives de haut niveau. Bien que non confortés par l'opinion actuelle, Frisch et McArthur [24] ont avancé la théorie d'une association entre la régularité menstruelle et le degré d'adiposité, suggérant qu'une proportion de graisses corporelles d'au moins $17 \%$ était capitale pour l'apparition des menstruations et le maintien de cycles normaux. Des données épidémiologiques récentes ont montré que 
l'âge à la ménarche était significativement $(\mathrm{p}<0,001)$ retardé chez des sportives que chez des témoins $(13,4 \pm 1,4$ contre 13,0 $\pm 1,3$ ans), et différait en fonction du type de sport, les sportives pratiquant des sports impliquant une minceur faisant état des retards les plus importants [10]. De ce fait, les activités préoccupantes sont celles comportant des aspects esthétiques et demandant une minceur telles que la gymnastique, le patinage artistique et la danse, ainsi que les sports où le contrôle pondéral est une stratégie intégrée de performance et tous ceux pour lesquels un entraînement physique intensif débute à un très jeune âge.

Des publications ont indiqué que les durées d'entraînement hebdomadaire de jeunes gymnastes artistiques en période prépubertaire et/ou en début de puberté s'élevaient à 36 heures/semaine [25]. Il n'est donc pas surprenant que certains auteurs aient considéré que les gymnastes féminines de haut niveau étaient exposées à un risque de retard de maturation $[23,26,27]$. Des études ont observé une réduction du potentiel de croissance et un retard de la ménarche chez des gymnastes artistiques $[4,8,26]$. Il existe toutefois des controverses considérables quant à un éventuel effet nocif d'un entraînement intensif, qui débute souvent avant la puberté, sur le potentiel de croissance des gymnastes artistiques de haut niveau. Les données longitudinales obtenues chez des gymnastes féminines et portant sur la fin de l'enfance et la période de l'adolescence sont limitées. Les résultats de deux études longitudinales à court terme ont suggéré que la poussée de croissance pubertaire des gymnastes était moins intense [26, 27]. Selon d'autres études, ce retard ne serait pas imputable à l'entraînement à la gymnastique lui-même, mais plutôt à des facteurs génétiques ou à des apports caloriques trop réduits pour satisfaire les demandes en énergie dues à la fois à la croissance et à l'entraînement [28, 29]. Une étude a suggéré que l'indice de masse corporelle était plus bas chez les gymnastes âgées de 7 à 10 ans, mais, selon une interprétation, cette différence a pu être due à la sélection de sportives minces [30]. Cette hypothèse est également confortée par une récente étude de Damsgaard et coll. [31] portant sur des données longitudinales indiquant que les différences individuelles de stature entre les pratiquants de divers sports étaient déjà apparentes à un âge précoce (2-4 ans), ce qui suggère une influence génétique. Les résultats d'une étude très récente sur la poussée de croissance à l'adolescence et l'âge squelettique de gymnastes féminines âgées de $8,7 \pm 1,5$ à 15,5 $\pm 1,5$ ans ont montré que ces sujets présentaient à l'adolescence une poussée de croissance portant sur la taille, la longueur estimée des membres inférieurs et la taille en position assise qui survenait environ 1 an plus tard et était légèrement moins intense que chez des jeunes filles non sportives [9]. L'âge à la ménarche et l'âge squelettique ont été compatibles avec une maturation somatique plus tardive. De plus, la poussée de croissance survenue lors de l'adolescence de ces gymnastes belges a été simi- laire à celle observée dans d'autres populations de jeunes filles de petite taille, normales et à maturation tardive, ou à maturation tardive et ayant des parents de petite taille [9]. En règle générale, la littérature montre que, bien qu'en moyenne les jeunes filles pratiquant la plupart des sports (par exemple nage, aviron, basket-ball, volley-ball et tennis) aient une stature identique ou supérieure à celle des médianes de référence pour la population normale, les gymnastes, les patineuses artistiques et les danseuses sont de plus petite taille et présentent constamment une masse corporelle plus légère, et que les gymnastes ont également des parents de plus petite taille que la moyenne [1].

Dans les activités à critères esthétiques telles que la gymnastique rythmique, un entraînement intensif débute habituellement bien avant la ménarche, et le succès est fortement influencé par la séduction visuelle et l'esthétique corporelle. Dès l'âge de 9 ans, il est souvent exigé des gymnastes rythmiques qu'elles atteignent des objectifs pondéraux irréalistes afin de parvenir à un aspect physique favorable ou de le conserver. Un retard de la ménarche, des irrégularités menstruelles et une faible adiposité ont été fréquemment observés chez des gymnastes rythmiques de haut niveau. Celles non encore réglées avaient été soumises à un entraînement de fréquence et de durée plus élevées et présentaient un plus bas indice de masse corporelle et d'adiposité relative comparativement à celles chez lesquelles la ménarche était survenue [11]. De plus, le développement mammaire était retardé chez des ballerines, qui ne présentaient que peu ou pas de développement des caractères sexuels secondaires (stade de Tanner 1 ou 2) à l'âge de 13 ans comparativement à des jeunes filles en bonne santé qui avaient habituellement atteint le stade 4 à la ménarche [32]. Un retard de la ménarche et du développement des seins a été observé chez des gymnastes adolescentes canadiennes de haut niveau comparativement à des témoins d'âge correspondant [33]. Le groupe mixte d'adolescentes pratiquant la gymnastique artistique ou rythmique incluses dans cette étude présentait des poids corporels significativement plus bas et une moindre adiposité relative comparativement à des participantes témoins normales. De même, le développement mammaire était plus tardif chez les gymnastes que chez les témoins (stades 2,7 \pm $0,2$ contre $3,8 \pm 0,1)$, mais la différence du développement de la pilosité pubienne entre les groupes n'atteignait pas la significativité (stades $3,1 \pm 0,2$ contre 4,0 $\pm 0,2$ ).

Selon une autre hypothèse, quand la puberté est temporairement interrompue sous l'effet de l'entraînement physique, la maturation de l'âge squelettique se réduit fréquemment tandis que la croissance se ralentit et que la concentration des stéroïdes gonadiques devient basse $[7,8]$. Malheureusement, seul un nombre très restreint d'études a apporté quelques informations sur le taux d'excrétion des stéroïdes gonadiques et/ou surrénaliens chez des jeunes filles actives en période péripubertaire. Malina et coll. [34] ont montré 
que l'activité des axes hypophyso-surrénaliens et hypophyso-gonadiques pouvait dépendre de la nature des exercices physiques. Dans le même domaine, Peltenburg et coll. [35] ont indiqué que des gymnastes en période prépubertaire présentaient de plus basses concentrations d'œstrone, de testostérone et d'androsténedione que des nageuses d'âge correspondant, tandis que les concentrations circulantes de $17 \beta$-estradiol, de sulfate de déhydroépiandrostérone (DHEA-S), de LH et de FSH étaient similaires entre ces deux groupes de sportives. En revanche, les concentrations de toutes ces hormones ne différaient pas entre des gymnastes et des nageuses en période pubertaire précoce [35]. Aucune différence n’a été observée entre un groupe de 56 gymnastes non réglées (s'entraînant 15-22 heures/semaine) et 53 témoins d'âge correspondant quant à la testostéronurie, la cortisolémie et la concentration de DHEA-S, tandis que le taux urinaire d'androsténedione était significativement plus bas chez les gymnastes que chez les témoins [36]. Comme attendu, une étude de Klentrou et coll. [33] a montré que les concentrations plasmatiques du $17 \beta$-estradiol et de la progestérone chez des gymnastes non réglées, dont la ménarche ultérieure avait été retardée, étaient similaires à celles mesurées chez des témoins au cours de la phase folliculaire, elles-mêmes significativement plus basses ( $\mathrm{p} \leq$ $0,05)$ que celles déterminées chez ces mêmes témoins pendant la phase lutéale.

\section{Entraînement sportif au cours de l'adolescence}

Un nombre croissant de filles suivent un entraînement sportif intensif à un jeune âge, et les domaines médicaux centrés sur la triade de la sportive sont donc devenus une préoccupation. Une augmentation du poids corporel peut négativement influencer les performances dans certaines activités, notamment les sports à critères esthétiques et à puissance élevée pour la masse corporelle [37]. En conséquence, la vie d'une adolescente sportive engagée dans ces activités comporte souvent une association d'entraînement physique intensif et de pressions destinées à l'obtention d'objectifs pondéraux irréalistes dans le but d'améliorer les performances et/ou l'aspect visuel. En conséquence, les tentatives de contrôle du poids et/ou d'obtention d'un aspect physique séduisant aboutissent souvent à une perturbation des profils alimentaires, qui a été associée à un retard de croissance et de maturation sexuelle chez des adolescentes sportives [38]. Les troubles alimentaires, la dysfonction menstruelle et l'ostéoporose représentent les trois composantes intercorrélées de la triade de la sportive, syndrome potentiellement grave souvent observé chez de jeunes filles et des femmes physiquement actives et pouvant résulter en une diminution des performances, en une morbidité à court et long terme et même en une mortalité [39].
Plus spécifiquement, les troubles alimentaires consistent en des comportements adoptés afin de perdre ou de contrôler le poids corporel ou d'obtenir une minceur ou une apparence esthétique [39]. Des publications ont indiqué que la prévalence de troubles alimentaires cliniques tels que l'anorexie mentale, la boulimie nerveuse et l'anorexie du sportif était significativement plus élevée chez des sportives que chez des témoins non actifs ou des sportifs de sexe masculin et chez des pratiquants d'activités sportives dépendant de la minceur et du poids comparativement à d'autres sports [40] En revanche, une étude de Fogelholm et Hiilloskorpi [41] menée chez 173 sportives finlandaises pratiquant divers sports a confirmé que, bien que le risque de troubles alimentaires puisse dépendre du type de sport, l'hypothèse selon laquelle certains groupes de sportives étaient exposés à un risque plus élevé que les témoins n’avait pas été examinée de façon adéquate. Chacun de ces comportements peut aboutir à des perturbations de la fonction menstruelle. Une incidence élevée d'aménorrhées secondaires a été décrite chez des sportives pratiquant certains sports; ces profils menstruels anormaux se sont avéré exercer un effet négatif sur la minéralisation osseuse et leurs conséquences à court et long terme pourraient donc être graves, notamment une augmentation de l'incidence des fractures de contrainte et l'ostéoporose [39, 42, 43]. Une sous-nutrition a été associée à des retards de croissance et de maturation sexuelle, et même à une infertilité $[38,42,44]$.

Dans le cadre d'une étude épidémiologique exhaustive, Torstveit et Sundgot-Borgen [45] ont déterminé les pourcentages de sportives de haut niveau et de sujets témoins exposés à un risque de triade de la sportive. Un questionnaire détaillé, qui comportait des questions sur les profils d'entraînement et/ou d'activité physique, les antécédents menstruels, la prise d'un contraceptif oral, les antécédents pondéraux, les profils alimentaires, les antécédents diététiques et les sous-échelles Body Dissatisfaction (insatisfaction corporelle) et Drive for Thinness (recherche de minceur) de l'Eating Disorder Inventory (inventaire des troubles alimentaires), a été rempli par une cohorte substantielle de sportives et de témoins non sportifs de sexe féminin. La batterie de questions a été soumise à la population totale des sportives de haut niveau en Norvège, représentant les équipes nationales junior et senior (âge: $13-39$ ans; $n=669$ ) et un groupe de témoins d'âge correspondant $(\mathrm{n}=607)$. Les résultats de l'étude ont été intéressants: (a) un pourcentage plus élevé de témoins $(69,2 \%)$ que de sportives $(60,4 \%)$ a été classé comme exposé à un risque de survenue de la triade de la sportive ( $\mathrm{p} \leq 0,01)$; (b) de même, un pourcentage plus élevé de témoins a fait état de l'utilisation de méthodes nocives de contrôle pondéral et (c) le score d'insatisfaction corporelle a été plus élevé chez les témoins ( $\mathrm{p} \leq 0,001)$. Toutefois, (a) une proportion plus élevée de sportives a fait état d'une dysfonction menstruelle et de fractures de contrainte 
comparativement aux témoins ( $\mathrm{p} \leq 0,05)$ et (b) les pourcentages de sportives pratiquant des sports impliquant une minceur $(70,1 \%)$ et de témoins $(69,2 \%)$ classés comme exposés à un risque de triade de la sportive ont été plus élevés que celui des sportives se consacrant à des sports où la minceur n'était pas un critère $(55,3 \%$; $p \leq 0,001)$ [45]. De plus, le pourcentage des participantes classées comme exposées à un risque de survenue de la triade a été plus élevé chez les sportives engagées dans des sports à critères esthétiques $(66,4 \%)$ que chez celles pratiquant des sports de balle $(52,6 \%)$ $(p \leq 0,001)$. Les auteurs ont conclu que les sportives pratiquant des sports où la minceur était un critère, ainsi que les femmes témoins non sportives, étaient plus exposées à un risque de survenue de la triade que les pratiquantes des sports où la minceur n'était pas nécessaire tels que les sports de balle [45]. Les résultats de l'étude norvégienne sont en faveur de l'hypothèse selon laquelle une proportion significative de sportives souffre d'une ou de plusieurs composantes de la triade $[45,46]$. Il est également important de souligner que les auteurs ont mentionné un risque significatif de survenue de la triade chez des femmes à activité physique normale. De ce fait, des programmes et outils éducatifs doivent être développés afin de favoriser la prévention de la triade et, de plus, ces programmes doivent s'adresser à toutes les jeunes filles et jeunes femmes physiquement actives [46].

\section{Irrégularités menstruelles au cours de la puberté}

Des irrégularités menstruelles, dont une aménorrhée primaire (absence de menstruations à l'âge de 16 ans), une aménorrhée secondaire (absence de menstruations pendant 3 mois ou plus après la ménarche) et une oligoménorrhée (durée du cycle menstruel supérieure à 40 jours), ont été observées chez des adolescentes sportives. La prévalence de «l'aménorrhée de la sportive» varie de 12 à $66 \%$ chez ces jeunes filles [47, 48]. Afin d'estimer l'influence d'un entrầnement intensif sur le cycle menstruel chez les sportives, Dusek [12] a examiné le moment de survenue de la ménarche et la prévalence des aménorrhées primaires et secondaires et des dysménorrhées chez 72 jeunes sportives actives (15-21 ans) et 96 témoins d'âge correspondant non engagés dans une activité sportive organisée ou régulière. L'échantillon des sportives incluait des joueuses de volley-ball et de basket-ball ainsi que des ballerines et des pratiquantes de sports de course. Les résultats ont montré que la prévalence des aménorrhées secondaires était trois fois plus élevée chez les sportives que chez les témoins $(\mathrm{p} \leq 0,05)$. Celle des aménorrhées primitives était substantiellement plus élevée chez les sportives que chez les témoins ( $\mathrm{p} \leq 0,05)$, mais celle des dysménorrhées était deux fois plus basse chez les premières que chez les secondes ( $\mathrm{p} \leq 0,001)$. La prévalence la plus éle- vée des aménorrhées secondaires a été enregistrée chez les coureuses, particulièrement de fond, tandis que seul un cas d'aménorrhée secondaire a été observé chez les joueuses de basket-ball [12]. Les coureuses de fond étaient également plus légères et plus minces que les témoins et les sportives pratiquant d'autres sports ( $\mathrm{p} \leq 0,05)$. Une étude précédente de Toriola [49] avait évalué la fonction menstruelle chez 155 sportives nigériennes (13-19 ans) appartenant à diverses catégories et 135 témoins (12-18 ans). Les menstruations étaient plus régulières chez les témoins (44\%) que chez les sportives (21\%), tandis que l'aménorrhée secondaire ou l'oligoménorrhée était plus prévalente chez les sportives (25\%) que chez les témoins (10\%). Quelle quait été l'activité sportive, le poids corporel des participantes présentant une oligoménorrhée était substantiellement plus faible par rapport à la stature, et ces jeunes femmes étaient également plus minces que celles classées dans d'autres catégories menstruelles [49]. Dans le groupe présentant une oligoménorrhée, une plus grande proportion de sportives (59\%) et de témoins $(57 \%)$ présentait une masse corporelle indiquant une minceur, se situant au-dessous du $41^{\text {ème }}$ percentile [49]. Les troubles menstruels ont été également mentionnés comme relativement plus fréquents chez les sportives ayant débuté leur entraînement avant la ménarche $[49,50]$.

Torstveit et Sundgot-Borgen [10] ont récemment examiné la prévalence des dysfonctions menstruelles dans la population totale des sportives norvégiennes de haut niveau $(n=669)$ représentant leur pays au niveau junior ou senior (à l'âge de 13-39 ans) et l'ont comparée à celle déterminée dans un échantillon témoin national d'âge correspondant $(\mathrm{n}=607)$. Un questionnaire détaillé a été remis à tous les sujets et comportait des questions sur les paramètres suivants: profil d'entraînement et/ou d'activité physique, antécédents menstruels, alimentaires et pondéraux, prise d'un contraceptif oral et inventaire des troubles alimentaires. Les résultats ont indiqué qu'un pourcentage plus élevé de sportives $(7,3 \%)$ que de témoins $(2,0 \%)$ avait fait état d'antécédents d'aménorrhée primaire ( $\mathrm{p} \leq 0,001)$. De plus, un pourcentage plus élevé de sportives engagées dans des sports impliquant une minceur ont fait état d'une dysfonction menstruelle en cours $(24,8 \%)$ comparativement à celles pratiquant des sports n'impliquant pas une minceur $(13,1 \%$; $p \leq 0,01)$ et aux témoins $(p \leq 0,05)[10]$. Ces données ont porté sur la quasi-totalité des sportives de haut niveau éligibles, et ont donc substantiellement étendu les études précédentes. Les auteurs ont suggéré que la différence de prévalence des dysfonctions menstruelles entre les sportives de haut niveau et les témoins n'était pas aussi importante que précédemment supposée, et que cette prévalence était plus basse chez les sportives et chez les témoins que celle déterminée voici une décennie [10]. Néanmoins, un pourcentage plus élevé de sportives pratiquant des sports impliquant une minceur et/ou un poids spécifique a fait état d'une dysfonc- 
tion menstruelle actuelle comparativement à celles exerçant des sports moins centrés sur ces facteurs [10].

Des études transversales menées chez des sportives aménorrhéiques ont montré des profils anormaux des hormones intervenant dans la reproduction, suggérant que la sécrétion de la GnRH par l'hypothalamus était perturbée et qu'en conséquence, une fonction hypothalamo-hypophysoovarienne normale ne s'instaurait pas, situation aboutissant à une diminution de la libération des gonadotrophines. Les concentrations de gonadotrophines et d'hormones ovariennes chez les sportives aménorrhéiques n’ont révélé aucune variation menstruelle en phase folliculaire et lutéale (signe d'inhibition ovarienne). Les concentrations d'estradiol et de progestérone en phase lutéale chez la seule gymnaste en phase de ménarche de l'étude de Klentrou et coll. [33] ont été plus basses que celles mesurées chez les témoins. D’autre part, les concentrations du $17 \beta$-estradiol décrites dans cette étude chez les sujets témoins n'ont pas atteint les valeurs adultes pour la phase folliculaire ou lutéale, mais ont été similaires à celles mentionnées pour des adolescentes, et les concentrations plasmatiques de la progestérone chez les témoins se sont situées dans les mêmes limites que celles décrites chez des adultes. Des études précédentes ont également indiqué de plus basses concentrations d'estradiol et de progestérone en phase lutéale chez des nageuses adolescentes comparativement à des témoins d'âge correspondant et des adultes, ce qui suggère que le corps jaune ne fonctionnait pas correctement chez les nageuses [51]. Une dysfonction ovulatoire a été également observée chez des sportives pratiquant des sports de compétition et exemptes d'irrégularités menstruelles, les résultats montrant que les concentrations de la progestérone étaient plus basses au cours de la phase lutéale [38]. Les causes suggérées de ces altérations ont inclus: (a) un hypercortisolisme (augmentation de la sécrétion d'hormone libérant la corticotrophine) induit par une restriction protéinoénergétique sévère aboutissant à une altération du profil épisodique normal de sécrétion de la LH $[47,52]$; (b) une hypoestrogénie dû à la disparition de la sécrétion pulsatile de la GnRH et (c) une insuffisance du mécanisme de rétrocontrôle positif des œstrogènes. Le mécanisme proposé à l'origine d'irrégularités menstruelles chez les nageuses a été une hyperandrogénie [14].

Au cours de la puberté, la maturation de l'axe hypothalamo-hypophysaire aboutit à une augmentation de la fréquence et de l'amplitude de la sécrétion pulsatile de la $\mathrm{GnRH}$, ce qui entraîne un accroissement de celles de la LH, de la FSH et des stéroïdes sexuels. La chronologie des pulsations est essentielle pour l'établissement d'un caractère cyclique ovulatoire normal. Le pic de LH survient la nuit au début de la puberté, et tout au long de la journée et de la nuit avec une amplitude nocturne plus basse à la fin de la puberté et à l'âge adulte [53]. Au cours des premières années suivant la ménarche, le rétrocontrôle hypothalamo-hypophyso-ovarien est instable en raison d'une augmentation de la LH basale et d'une chronologie «immature» de la sécrétion pulsatile de la $\mathrm{LH}$. Il en résulte des cycles menstruels irréguliers, une hyperandrogénie ovarienne, une multifollicularité et une anovulation [54]. De ce fait, des cycles peu fréquents, irréguliers et anovulatoires, des saignements utérins anormaux et une dysménorrhée sont fréquents au cours des premières années suivant la ménarche $[2,3]$. Pendant le développement pubertaire normal, un cycle peut être considéré comme normal si sa longueur est de 21-40 jours, si les pertes sanguines sont de $20-80 \mathrm{ml}$ et si la durée des menstruations est de 2-8 jours [2,3]. L'étape finale de la maturation est l'instauration d'un rétrocontrôle œstrogénique positif s'exerçant sur l'hypophyse et l'hypothalamus et stimulant l'apparition en milieu de cycle du pic de $\mathrm{LH}$ nécessaire à l'ovulation. Labsence de rétrocontrôle œstrogénique positif au cours de la première année suivant la ménarche résulte en des cycles anovulatoires et peut également traduire une étape transitoire normale de l'hyperandrogénie ovarienne [3]. De plus, les cycles ovulatoires de l'adolescente se caractérisent par une longue phase folliculaire, une ovulation tardive et une courte phase lutéale insuffisante. La sélection d'un follicule dominant est irrégulière et le follicule développé présente un diamètre plus petit que chez une femme adulte [55]. Toutes ces irrégularités disparaissent habituellement avec le début de l'ovulation régulière $[2,3]$. De ce fait, l'aménorrhée de la sportive peut être simplement un signe de puberté tardive ou de troubles induits par une restriction calorique affectant l'axe hypothalamo-hypophyso-ovarien.

\section{Rôle de la restriction calorique}

Dans le contexte de cet article, les troubles alimentaires peuvent être utilisés comme synonymes de sous-nutrition. Comme mentionné plus haut, une préoccupation médicale majeure concernant les jeunes sportives de haut niveau est l'insuffisance de leurs apports caloriques par rapport à la grande quantité d'énergie dépensée au cours de l'entraînement quotidien. L'association d'exercices physiques intensifs à une sous-nutrition exerce d'importants effets sur la libération des gonadotrophines, l'activation de l'axe gonadique, la chronologie de la puberté et l'apparition de troubles menstruels chez les adolescentes sportives [32, 44]. De plus, des formes moins sévères de restriction alimentaire par rapport à l'énergie libérée ont été associées à des phases lutéales inadéquates et à une anovulation ainsi qu'à des pics anormaux de LH et de FSH au cours du cycle menstruel [38].

Le processus naturel du cycle menstruel est régulé par les interactions complexes des hormones ovariennes. Une activité physique intensive altère la concentration de plusieurs de ces hormones. Ces altérations peuvent également avoir 
Fig. 1. Représentation schématique des contrôles nutritionnels de la physiologie de la reproduction chez la femme. Modifiée d'après Wade et Jones [60].

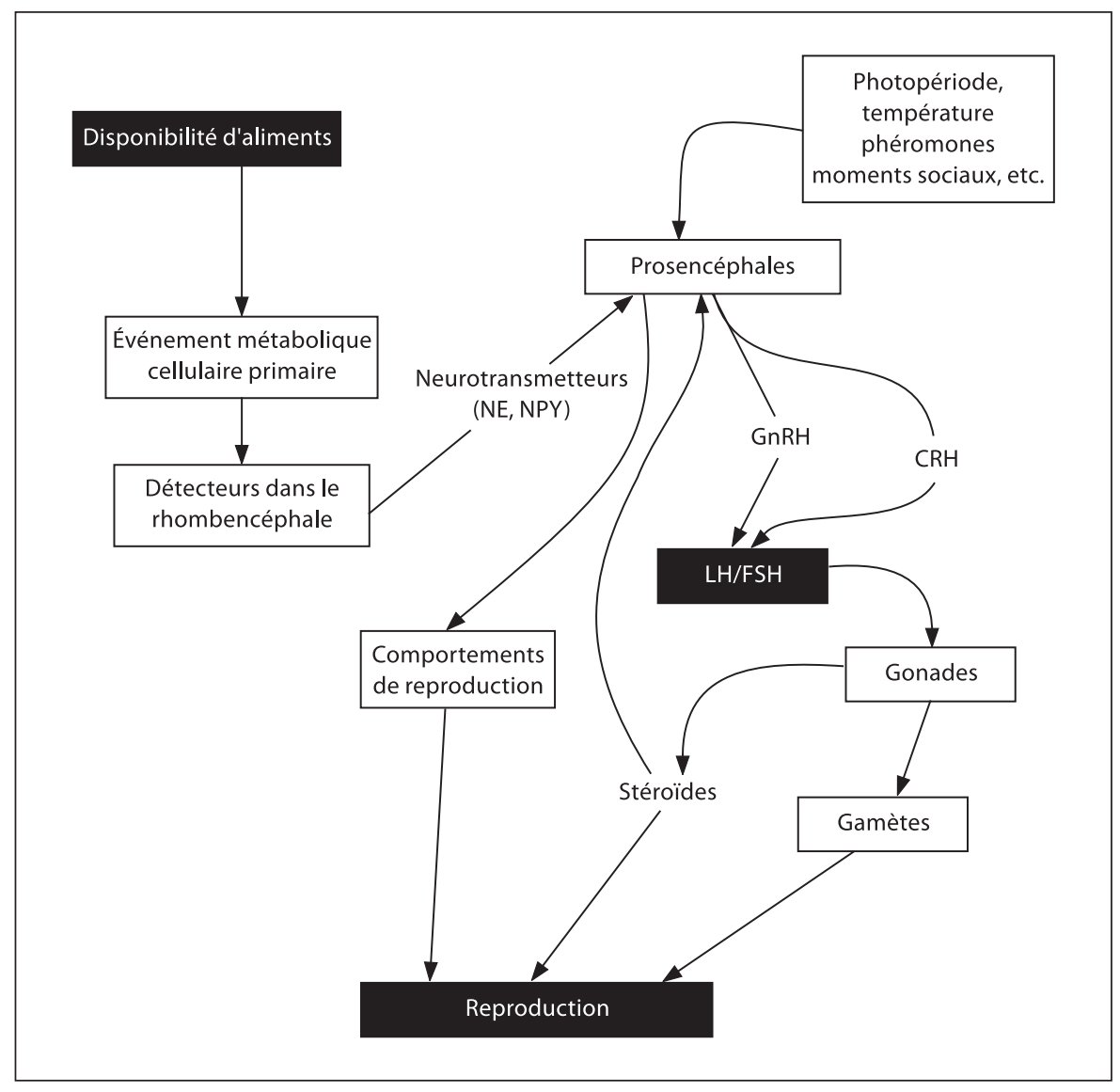

des implications pour l'utilisation des substrats au cours de l'effort. Zderic et coll. [56] ont souligné que, chez des sportives correctement nourries, l'estradiol médiait les modifications du métabolisme des glucides et des lipides. De ce fait, l'oxydation de ces substances peut être régulée de façon différente sur l'ensemble du cycle menstruel en raison des importantes modifications des hormones ovariennes circulantes. Quand l'estradiol est élevé au cours de la phase lutéale, la production et la concentration de l'acide lactique diminuent et le recours à l'oxydation des lipides est plus élevé. Il en est ainsi chez la femme quand les lactates diminuent, quelle que soit l'intensité de l'exercice, ce qui suggère que l'utilisation des lipides pourrait être un effet direct de la concentration élevée de l'estradiol [5]. Il existe une association manifeste et directe entre l'utilisation des substrats énergétiques et la fonction reproductive, mais il reste à savoir s'il existe également une interaction entre la disponibilité de ces substrats et la reproduction.

Comme précédemment mentionné, des auteurs ont soutenu que les femmes devaient atteindre un degré minimal d'adiposité afin de franchir la puberté et de maintenir un cycle normal [24]. Cette "hypothèse de la graisse critique» a été souvent mentionnée de nombreuses façons et a été acceptée par les cliniciens. Toutefois, selon de nouvelles publications, il n'existe en fait aucune association directe de cause à effet entre adiposité et fonction reproductrice et, bien qu'elles tendent à être intercorrélées, l'adiposité et l'aménorrhée n'ont pas été reliées de façon causale [57-60]. Au contraire, une nouvelle théorie, l'hypothèse des substrats énergétiques métaboliques, a été développée et suppose que la disponibilité de l'énergie, et non la graisse corporelle, régule la fonction reproductrice chez la femme. En résumé, cette hypothèse suggère que la fonction reproductrice, comme la consommation alimentaire, répond à des modifications à court terme de l'oxydation des substrats énergétiques métaboliques. La sécrétion de la LH, et par inférence celle de la $\mathrm{GnRH}$, répondent presque instantanément à des modifications subites de l'oxydation de ces substrats [61]. Des détecteurs de substrats énergétiques situés dans le rhombencéphale paraissent participer au contrôle de la libération de la LH. Quand le rhombencéphale «viscéral» détecte les informations concernant la disponibilité de subs- 
trats énergétiques, il envoie des projections directes et indirectes à des régions du prosencéphale dont les rôles importants dans la régulation de l'équilibre énergétique et de la fonction reproductrice sont connus (fig. 1). Des projections contenant à la fois des catécholamines et le neuropeptide $\mathrm{Y}$ ont été identifiées comme participant à cette voie [60]. De plus, des projections contenant des catécholamines et le neuropeptide $\mathrm{Y}$ aboutissant au prosencéphale peuvent également stimuler la sécrétion d'hormones libérant la corticotrophine, ce qui aboutit à un hypercortisolisme, dont le rôle prédominant dans la suppression de la sécrétion pulsatile de la LH au cours d'une sous-nutrition chez de jeunes sportives avait été précédemment suggéré $[47,52]$. Des recherches significativement plus nombreuses sont toutefois nécessaires afin d'explorer davantage cette interaction entre les modifications à court terme de la disponibilité des substrats énergétiques et l'aménorrhée de la sportive, particulièrement celles concernant la puberté.

\section{Conclusions}

Dans le cadre des activités sportives, l'instauration d'un entraînement physique intensif à un très jeune âge exerce une contrainte significative sur l'organisme au cours d'une période critique du développement, particulièrement chez les jeunes filles. L'association d'exercices intensifs à une sous-nutrition peut influencer le début de la puberté et la maturation sexuelle chez les sportives, particulièrement celles qui pratiquent des sports où le contrôle du poids (afin de tenter d'entrer dans une classe de poids ou de maximiser le rapport puissance/poids) ou l'aspect esthétique est une stratégie intégrée de performance ou une composante capitale de la réussite.

Certaines études ont suggéré qu'un retard de la ménarche pouvait être dû à des facteurs génétiques, mais des données considérables indiquent que des facteurs environnementaux jouent également un rôle significatif. En raison des nombreux facteurs précédemment démontrés comme influençant la ménarche, le rôle de l'entraînement physique comme seul facteur causal dans le retard d'apparition de la puberté chez les jeunes filles sportives est encore mal défini. Une prévalence plus élevée des dysfonctions menstruelles a été décrite chez des adolescentes pratiquant des sports impliquant une minceur et une limitation du poids comparativement à d'autres activités sportives. Les recherches disponibles plaident en faveur du rôle de facteurs liés à la nutrition et au bilan énergétique, ainsi que du moment de l'instauration d'un entraînement intensif, dans l'étiologie des irrégularités menstruelles associées aux activités physiques chez les sportives en période pubertaire. Les études futures devront être longitudinales afin d'explorer les effets à long terme de l'entraînement sportif et d'examiner le rôle de la disponibilité et de l'utilisation des substrats énergétiques dans la croissance et la maturation des jeunes femmes.

\section{Références}

1 Baxter-Jones ADG, Maffulli N: Intensive training in elite young female athletes. $\mathrm{Br} \mathrm{J}$ Sports Med 2002;36:13-15.

$\checkmark 2$ Caufriez A: Menstrual disorders in adolescence: pathophysiology and treatment. Horm Res 1991;36:156-159.

3 Hamm T: Physiology of normal female bleeding. NAACOGS Clin Issu Perinat Womens Health Nurs 1991;2:289-293.

-4 Lindholm C, Hagenfeldt K, Rangertz B: Pubertal development in elite juvenile gymnasts. Effects of physical training. Acta Obstet Gynecol Scand 1994;73:269-273.

5 Bunt JC: Metabolic actions of estradiol: significance for acute and chronic exercise responses. Med Sci Sports Exerc 1990;22:286290.

-6 Mansfield M, Emans J: Growth in female gymnasts: should training decrease during puberty? J. Pediatr 1993;122:237-240.

7 Theintz GE: Endocrine adaptation to intensive physical training during growth. Clin Endocrinol 1994;41:267-272.
8 Theintz GE, Ladame F, Howald $\mathrm{H}$, et al: L'enfant, la croissance et le sport de haut niveau. Schweiz Z Med Traumatol 1994;3: 7-15.

9 Thomis M, Claessens AL, Lefevre J, Philippaerts R, Beunen GP, Malina RM: Adolescent growth spurts in female gymnasts. J Pediatr 2005;146:239-244.

10 Torstveit MK, Sundgot-Borgen J: Participation in leanness sports but not training volume is associated with menstrual dysfunction: a national survey of 1276 elite athletes and controls. Br J Sports Med 2005;39:141147.

11 Klentrou P, Plyley M: Onset of puberty, menstrual frequency, and body fat in elite rhythmic gymnasts compared with normal controls. Br J Sports Med 2003;37:490-494.

12 Dusek T: Influence of high intensity training on menstrual cycle disorders in athletes. Croat Med J 2001;42:79-82.
13 Pigeon P, Oliver I, Charlet JP, Rochiccioli $\mathrm{P}$ : Intensive dance practice: repercussions on growth and puberty. Am J Sports Med 1997;25:243-247.

14 Constantini NW, Warren MP: Menstrual dysfunction in swimmers: a distinct entity. J Clin Endocrinol Metab 1995;80:27402744.

15 Malina RM, Ryan RC, Bonci CM: Age at menarche in athletes and their mothers and sisters. Ann Hum Biol 1994;21:417-422.

16 Hata E, Aoki K: Age at menarche and selected menstrual characteristics in young Japanese athletes. Res Q Exerc Sport 1990;61: 178-183.

17 Calabrese LH: Nutritional and medical aspects of gymnastics. Clin Sports Med 1985; 4:23-30.

18 Calabrese LH, Kirkendall DT, Floyd M: Menstrual abnormalities, nutritional patterns, and body composition in female classical ballet dancers. Phys Sportsmed 1983;11: 86-98. 
19 Malina R: Menarche in athletes: a synthesis and hypothesis. Ann Hum Biol 1973;10:124.

20 Baxter-Jones ADG, Helms PJ: Effects of training at a young age: a review of the training of young athletes (TOYA) study. Pediatr Exerc Sci 1996;8:310-327.

-21 Kaprio J, Rimpela A, Winter T, Viken RJ, Rimpela M, Rose RJ: Common genetic influences on BMI and age at menarche. Hum Biol 1995;67:739-753.

22 Malina RM, Waynarowska B, Bielicki T, et al: Prospective and retrospective longitudinal studies of the growth, maturation and fitness of Polish youth active in sports. Int J Sports Med 1997;18(suppl):S179-S185.

-23 Frisch R, Gotz-Welbergen AV, McArthur JW: Delayed menarche and amenorrhea of college athletes in relation to age of onset of training. JAMA 1981;246:1559-1563.

-24 Frisch RE, McArthur JW: Menstrual cycles: Fatness as a determinant of minimum weight for height necessary for their maintenance or onset. Science 1974;185:949-951.

-25 Bass, S, Pearce, G, Bradney, M, et al: Exercise before puberty may confer residual benefits in bone density in adulthood: studies in active prepubertal and retired female gymnasts. J Bone Miner Res 1998;13:500-507.

-26 Theintz GE, Howald H, Weiss U, et al: Evidence for a reduction of growth potential in adolescent female gymnasts. J Pediatr 1993; 122:306-313.

27 Bass S, Bradney M, Pearce G, et al: Short stature and delayed puberty in gymnasts: influence of selection bias on leg length and the duration of training on trunk length. J Pediatr 200;136:149-155.

28 Ledoux M, Brisson G, Peronnet F: Nutritional habits of young female gymnasts. Med Sci Sports Exerc 1983;14:145.

29 Ersoy G: Dietary status and anthropometric assessment of child gymnasts. J Sports Med Phys Fitness 1991;31:577-580.

- 30 Benardot D, Czerwinski C: Selected body composition and growth measures of junior elite gymnasts. J Am Diet Assoc 1991;91:2933.

31 Damsgaard R, Bencke J, Matthiesen G, et al: Is pubertal growth adversely affected by sport? Med Sci Sports Exerc 2000;32:16981703.

32 Warren MP: The effects of exercise on pubertal progression and reproductive function in girls. J Clin Endocrinol Metab 1980; 51:1150-1157.

33 Klentrou P, Flouris AD, Plyley M: Pubertal maturation, hormonal levels and body composition in Canadian male and female gymnasts; in Marfell-Jones M, Stewart A, Gordon R, Olds T (eds): Kinanthropometry IX. Proc 9th Int Conf International Society for the Advancement of Kinanthropometry (ISAK). London, Routledge, 2006, in press.
34 Malina RM, Waneen W, Spirduso CT, Baylor A: Age at menarche and selected menstrual characteristics in athletes at different competitive levels and in different sports. Med Sci Sports 1978;10:218-222.

- 35 Peltenburg AL, Erich WBM, Thijssen JJH, et al: Sex hormone profiles of premenarchea athletes. Eur J Appl Physiol 1984;52:385392.

- 36 Jaffré C, Lac G, Benhamou CL, Courteix D: Effects of chronic intensive training on androgenic and cortisol profiles in premenarcheal female gymnasts. Eur J Appl Physiol 2002;87:85-89.

37 Wilmore JH: Body weight standards and athletic performance; in Brownell KD, Rodin J, Wilmore JH (eds): Eating, Body Weight and Performance in Athletes: Disorders of Modern Society. Philadelphia, Lea \& Febiger, 1992, pp 315-329.

38 Loucks AB, Vaitukaitis J, Cameron JL, et al: The reproductive system and exercise in women. Med Sci Sports Exerc 1992;24 (suppl):S288-S293.

39 Yeager KK, Agostini R, Nattiv A, et al: The female athlete triad. Med Sci Sports Exerc 1993;25:775-777.

40 Sundgot-Borgen J, Torstveit MK: Prevalence of eating disorders in elite athletes is higher than in the general population. Clin J Sport Med 2004;14:25-32.

41 Fogelholm M, Hiilloskorpi H: Weight and diet concerns in Finnish female and male athletes. Med Sci Sports Exerc 1999;31:229_ 235.

42 Drinkwater BL, Nilson K, Chesnut CH, et al: Bone mineral content of amenorrheic and eumenorrheic athletes. N Engl J Med 1984; 311:277-281.

43 DiFiori JP: Menstrual dysfunction in athletes. How to identify and treat patients at risk for skeletal injury. Postgrad Med 1995; 97:143-156.

44 Loucks AB, Mortola JF, Girton L, et al: Alterations in the hypothalamic-pituitaryovarian and the hypothalamic-pituitaryadrenal axes in athletic women. J Clin Endocrinol Metab 1989;68:402-411.

45 Torstveit MK, Sundgot-Borgen J: The female athlete triad: are elite athletes at increased risk? Med Sci Sports Exerc 2005;37:184193.

46 Torstveit MK, Sundgot-Borgen J: The female athlete triad exists in both elite athletes and controls. Med Sci Sports Exerc 2005;37: 1449-1459.
47 Constantini NW, Warren MP: Special problems of the female athlete. Baillieres Clin Rheumatol 1994;8:199-219.

48 Patterson DF: Menstrual dysfunction in athletes: assessment and treatment. Pediatr Nurs 1995;21:227-229, 310.

49 Toriola AL: Survey of menstrual function in young Nigerian athletes. Int J Sports Med 1988;9:29-34.

50 Toriola AL, Mathur DN: Menstrual dysfunction in Nigerian athletes. Br J Obstet Gynaecol 1986;93:979-985.

51 Bonen A, Belcastro AN, Ling WY, et al: Profiles of selected hormones during menstrual cycles of teenage athletes. J Appl Physiol 1981;50:545-551.

52 Fichter MM, Pirke KM: Effect of experimental and pathological weight loss upon the hypothalamo-pituitary-adrenal axis. Psychoneuroendocrinology 1986;11:295-305.

53 Wenninck JMB, Delemarre-Van De Waal HA, Shoemaker R, Shoemaker H, Shoemaker J: Luteinizing hormone and follicle stimulating hormone secretion patterns in girls throughout puberty measured using highly sensitive immunoradiometric assays. Clin Endocrinol 1990;33:333-344.

54 Venturoli S, Porcu E, Fabbri R, et al: Ovarian multifollicularity, high LH and androgen plasma levels, and anovulation are frequent and strongly linked in adolescent irregular cycles. Acta Endocrinol 1986;111:368-372.

55 Apter D, Räisänen I, Ylöstalo PRV: Follicular growth in relation to serum hormonal patterns in adolescent compared with adult menstrual cycle. Fertil Steril 1987;47:82-88.

56 Zderic TW, Coggan AR, Ruby BC: Glucose kinetics and substrate oxidation during exercise in the follicular and luteal phases. J Appl Physiol 2001;90:447-453.

57 Bronson FH, Manning JM: The energetic regulation of ovulation: a realistic role for body fat. Biol Reprod 1991;44:945-950.

58 Loucks AB, Verdun M, Heath EM: Low energy availability, not stress of exercise, alters LH pulsatility in exercising women. J Clin Endocrinol Metab 1998;84:37-46.

59 Loucks AB: Energy availability, not body fatness, regulates reproductive function in women. Exerc Sports Sci Rev 2003;31:144148 .

60 Wade GE, Jones JE: Lessons from experimental disruption of estrous cycles and behaviors. Med Sci Sports Exerc 2003;35:15731580

61 Schneider JE, Wade GN: Inhibition of reproduction in service of energy balance; in Wallen K, Schneider JE (eds): Reproduction in Context. Cambridge, MIT Press, 1999, pp $35-82$. 